\title{
Contagious agalactia monitoring in caprine herds through regular bulk tank milk sampling
}

\author{
F. Tardy,,${ }^{1,2 *}$ M. Treilles, ${ }^{3}$ E. Gay, ${ }^{4}$ C. Ambroset, ${ }^{1,2}$ A. Tricot, ${ }^{1,2}$ C. Maingourd, ${ }^{3}$ J. Vialard, ${ }^{5}$ and D. Le Grand ${ }^{1,2}$ \\ ${ }^{1}$ Laboratoire de Lyon, UMR Mycoplasmoses des Ruminants, ANSES, Université de Lyon, F-69364, Lyon, France \\ ${ }^{2}$ VetAgro Sup, UMR Mycoplasmoses des Ruminants, Université de Lyon, F-69280, Marcy-L'étoile, France \\ ${ }^{3}$ Laboratoire QUALYSE, ZAE Montplaisir, F-79220 Champdeniers-St Denis, France \\ ${ }^{4}$ Laboratoire de Lyon, Unité Épidémiologie et appui à la Surveillance, ANSES, Université de Lyon, F-69364, Lyon, France \\ ${ }^{5}$ Laboratoire de Niort, ANSES, F-79024, Niort, France
}

\section{ABSTRACT}

Surveillance and control of Mycoplasma spp. responsible for contagious agalactia (CA) in caprine herds are important challenges in countries with a large smallruminant dairy industry. In the absence of any clinical signs, being able to determine the potential circulation of mycoplasmas within a herd could help to prevent biosecurity issues during animal exchanges between farms and improve health management practices. The objective of this study was to determine whether regular sampling of bulk tank milk was suitable for such surveillance. Twenty farms were sampled once a month for $2 \mathrm{yr}$ and CA-responsible mycoplasmas were detected by real-time PCR on DNA extracted from milk, using 3 different DNA extraction methods. The pattern of mycoplasma excretion in bulk tank milk was assessed over time and several herd characteristics were recorded together with any event occurring within the herds. In general, the results obtained with the different detection methods were comparable and mainly agreed with the culture results. Several patterns of excretion were observed but were not related to herd characteristics (size, breed, and so on). Recurrence of the same (sub)species and same pulsed-field gel electrophoresis subtype during the 2-yr period is indicative of the considerable persistence of mycoplasmas. This persistence was associated with intermittent excretion. In conclusion, bulk tank milk sampling could be valuable for controlling $\mathrm{CA}$ in caprine herds provided it is repeated several times, yet to be defined, per year and analyzed using an appropriate methodology and the right cut-off for interpretation.

Received November 1, 2018.

Accepted February 21, 2019.

*Corresponding author: florence.tardy@anses.fr
Key words: caprine contagious agalactia, bulk tank milk monitoring, Mycoplasma spp., excretion, persistence

\section{INTRODUCTION}

Mycoplasmosis surveillance and control in caprine herds is an important challenge in France and in other countries with a large small-ruminant dairy industry. Contagious agalactia (CA), the principal mycoplasmosis in goats, is a syndrome apparent as various clinical signs (pneumonia, mastitis, arthritis, and keratoconjunctivitis) in young animals and adults. It is associated with 4 causative agents, namely Mycoplasma agalactiae, Mycoplasma putrefaciens, Mycoplasma mycoides ssp. capri, and Mycoplasma capricolum ssp. capricolum (Corrales et al., 2007; Gomez-Martin et al., 2013). Contagious agalactia is distributed worldwide, the main enzootic areas being Southern Europe, the Middle East, Asia, and North Africa, and is classified as notifiable by the OIE (World Organization for Animal Health). It often responds poorly to antimicrobial treatments, despite the overall in vitro susceptibility of its causative agents (Poumarat et al., 2016; Prats-van der Ham et al., 2018), and has a significant adverse effect on the farming economy (Gomez-Martin et al., 2013). Furthermore, the efficacy of commercial or experimental vaccines has yet to be demonstrated, as only a few studies have been dedicated to their evaluation (Nicholas et al., 2008; Agnone et al., 2013).

The infection is most often initiated within a herd by bringing in animals from another farm that are clinically healthy but asymptomatically carry mycoplasmas (Bergonier and Poumarat, 1996; Al-Momani et al., 2008). Testing individual animals before their introduction is unfortunately impossible for both economic and technical reasons. Indeed, no commercial ELISA tests are available, except for $M$. agalactiae, and due to the existence of several potential body niches of asymptomatic carriage, including the external ear canal (Tardy et 
al., 2007; Gomez-Martin et al., 2012), direct diagnosis would require taking several samples from a single animal, which would be too expensive.

Consequently, being able to determine the potential circulation of mycoplasmas within a herd could help to minimize biosecurity issues during animal exchanges between farms and improve health management practices (separation of young, cleaning of milking utensils, milking order, and so on).

We hypothesize that regularly sampling bulk tank milk (BTM) could be appropriate to detect mycoplasmas within a herd and to try and establish a herd status. As a first step to validate this hypothesis, the present study was conducted to determine the pattern of mycoplasma excretion in BTM over time in 20 farms sampled once a month for 2 yr. Mycoplasmas responsible for CA were detected by real-time PCR (rtPCR) and 3 DNA extraction methods from milk were compared for their overall operational performance. The persistence and diversity of Mycoplasma (sub)species and isolates within a herd were monitored.

\section{MATERIALS AND METHODS}

\section{Herds Selection, Health Monitoring, and Milk Sampling}

Twenty herds were included in the study (Supplemental Table S1; https://doi.org/10.3168/jds.2018-15889). Each had shown at least one BTM sample positive for mycoplasmas just before the study or during the months (5 to 11) preceding the study, evidenced by real-time PCR diagnosis (LSI Vetmax Triplex Contagious Agalactia - MA \& MM, Thermo Fisher Scientific, Villebon sur Yvette, France) or a clinical episode of mycoplasmosis (or both) in the months or years preceding the study, diagnosed through the surveillance network Vigimyc (Poumarat et al., 2014). The herds were located in 2 main goat breeding areas of France, namely Poitou-Charentes-Vendée $(\mathbf{P C}, \mathrm{n}=10)$ and Rhône-Alpes (RA, n = 10). Breeding was either enclosed [i.e., without a grazing area (10/10 of PC farms and $4 / 10$ of RA farms)] or pasture-based (6/10 RA farms). Goat farms ranged in size from 240 to 1,800 animals in the PC region and from 144 to 400 animals in the RA region with a mean milk production per goat and per year ranging from 750 to $1,000 \mathrm{~kg}$ and from 380 to $950 \mathrm{~kg}$ in $\mathrm{PC}$ and $\mathrm{RA}$, respectively.

The study was conducted over 2 yr (April 2014 to April 2016). A BTM sample was taken monthly from each farm, and at the same time, any change in housing conditions, husbandry, and the farm environment that could interfere with mycoplasma excretion was recorded using a detailed questionnaire. This included the entry/ exit of animals as well as changes in feedlot composition (size, mixing, and so on) or livestock production (delivering, lactation and drying period, reproduction, batch changes, building sanitization, food changes, and so on), or both.

Whatever the size of the herd, milk was sampled directly from the main tank under stirring. The milk samples (30 to $50 \mathrm{~mL}$ ) were placed in cool boxes and transported directly to the laboratory. FTA Elute cards (GE Healthcare Life Sciences, Vélizy-Villacoublay, France) were spotted immediately upon arrival in the laboratory. The rest of the milk samples were (1) stored at $4^{\circ} \mathrm{C}$, a temperature shown to ensure a stable M. agalactiae and M. mycoides ssp. capri viable count (Amores et al., 2010) before being processed, (2) processed within 24 to $48 \mathrm{~h}$, and then (3) frozen at $-80^{\circ} \mathrm{C}$ before possible further investigation using method M2 (see hereafter).

\section{DNA Extraction and rtPCR Screening}

Three DNA extraction methods were assessed. In the first method (M1), DNA was directly extracted from $0.2 \mathrm{~mL}$ of fresh milk. This method included a lysis step, where milk was incubated for $30 \mathrm{~min}$ at $70^{\circ} \mathrm{C}$ with proteinase $\mathrm{K}$, and an extraction/purification step using a commercial platform based on magnetic-bead technology (KingFisher Flex Instruments, Thermo Fisher Scientific). The second method (M2) was conducted on frozen milk and consisted of preamplification of the mycoplasmal target with culture of $200 \mu \mathrm{L}$ of milk in $1.8 \mathrm{~mL}$ of broth medium followed by a bulk DNA extraction technique adapted from Ariza-Miguel et al. (2012). In brief, $100 \mu \mathrm{L}$ of a 2 -d preculture was added to $900 \mu \mathrm{L}$ of sterile PBS and centrifuged for $15 \mathrm{~min}$ at $15,000 \times g\left(4^{\circ} \mathrm{C}\right)$. The pellet was resuspended in $10 \mathrm{mM}$ Tris ( $\mathrm{pH} 8.0)$ and incubated on a shaker (500 rpm) for $20 \mathrm{~min}$ at $95^{\circ} \mathrm{C}$. After $3 \mathrm{~min}$ of centrifugation at 15,000 $\times g\left(4^{\circ} \mathrm{C}\right)$, the supernatant was transferred to a new tube and used for rtPCR analysis $(5 \mu \mathrm{L})$. The only method used on samples from both RA and PC areas was M2. The third method (M3) consisted of spotting $0.2 \mathrm{~mL}$ of milk on each spot of a FTA Elute card from which DNA was extracted using a high-speed benchtop homogenizer (FastPrep-24, MP Biomedicals, Illkirch, France). In brief, one whole spot of the FTA membrane was roughly cut and added to a bead beating tube (Lysing Matrix E 2-mL tubes, MP Biomedicals) containing 1.4-mm ceramic spheres, 0.1-mm silica spheres, and a single $4-\mathrm{mm}$ glass bead. After adding $1.5 \mathrm{~mL}$ of molecular biology quality water, the tubes were shaken for $40 \mathrm{~s}$ at 6,000 rpm in the FastPrep homogenizer. 
They were then incubated at $100^{\circ} \mathrm{C}$ for $20 \mathrm{~min}$, then centrifuged for $1 \mathrm{~min}$ at $10,000 \times g$ at room temperature. Five microliters of the DNA extracts, contained in the supernatant, was used for the PCR assays.

Each DNA sample was subjected to the same multiplex rtPCR assay (LSI Vetmax Triplex Contagious Agalactia - MA \& MM, Thermo Fisher Scientific), using a commercial kit that was developed some years ago (Becker et al., 2012). This rtPCR assay is able to detect both $M$. agalactiae on the one hand and also on the other hand 3 (sub)species of the M. mycoides cluster (namely $M$. mycoides ssp. capri, $M$. capricolum ssp. capricolum, and $M$. putrefaciens), without individual discrimination.

The rtPCR assay was run either on a Light Cycler 480 platform from Roche (M2) or a Biorad CFX96 rtPCR detection system (M1 and M3). As first line analysis, samples with cycle threshold $(\mathbf{C t})>40$ were interpreted as negative for both Taqman probes, VIC (2'-chloro-7'phenyl-1,4-dichloro-6-carboxy-fluorescein) for $M$. agalactiae, and FAM (6-carboxyfluorescein) for (sub)species of the cluster M. mycoides. Detailed comparative analysis of the positive results obtained with culture and the different extraction methods then prompted us to refine this cut-off.

\section{Mycoplasma Isolate Identification and Pulsed-Field Gel Electrophoresis Subtyping}

Pre-cultures of milks that were rtPCR-positive with M2 were sub-cultured on agar plates to isolate the corresponding mycoplasmas. The isolation and subsequent identification of isolates was considered as a standard reference test (Nicholas and Baker, 1998) to confirm, deny, or refine any rtPCR positive results obtained with M2. It was not run when the PCR results were negative and hence could not be used as a gold standard. Mycoplasma strains were grown at $37^{\circ} \mathrm{C}$ under $5 \% \mathrm{CO}_{2}$ in PPLO broth supplemented as detailed previously (Poumarat et al., 1991) and purchased from Indicia Production (Saint Genis l'Argentière, France). The isolates were identified by membrane-filtration immunobinding tests, MF-Dot (Poumarat et al., 1991).

Some representative isolates were then further subtyped by pulsed-field gel electrophoresis (PFGE), as previously described (Tardy et al., 2007), using the enzyme BamHI for M. capricolum ssp. capricolum and M. mycoides ssp. capri, and MluI for M. putrefaciens and $M$. agalactiae. Images were analyzed with the software GelCompar II v6.6 created by Applied Maths NV (Sint-Martens-Latem, Belgium). The similarity analysis was carried out using the Dice coefficient (position tolerance, $1.5 \%$ ) and a dendrogram was constructed by unweighted pair group method with arithmetic mean.

\section{Statistical Tests}

The 2 by 2 agreement of the 3 methods for the 2 Mycoplasma targets (M. mycoides cluster and M. agalactiae) was investigated by calculating the observed agreement and Cohen's kappa coefficient on the binomial results (positive/negative, a $\mathrm{Ct}>40$ was used for negative sample categorization) of the rtPCR. This coefficient is considered more robust than the simple observed agreement calculation because it takes the hypothetical probability of agreement by chance $(50 \%)$ into account. The kappa coefficient is classically interpreted as (1) no agreement (except what would be expected by chance) if kappa is less than 0, (2) slight agreement if 0 to 0.2 , (3) fair agreement if 0.2 to $0.4,(4)$ moderate agreement if 0.4 to $0.6,(5)$ substantial agreement if 0.6 to 0.8 , and (6) almost perfect agreement if the kappa value is 0.8 to 1.0 (Landis and Koch, 1977).

\section{RESULTS AND DISCUSSION}

\section{Comparison of Methods for Mycoplasma Detection from BTM Samples}

A total of 465 milk samples were collected and 418 were analyzed by M2. Out of these, 210 were also analyzed by M1 and 233 by M3. The kappa coefficient calculations between the rtPCR methods are summarized in Table 1. In brief, rtPCR analyses conducted on DNA extracted directly from fresh milk (M1), from frozen milk with enrichment (M2), or from FTA Elute card (M3) yielded almost perfect agreement for $M$. agalactiae (97.6 to 99.0\%), and to a lesser extent, for (sub)species of the M. mycoides cluster (91.0 to $93.3 \%$ ). It should be noted that when discordant pairs were examined, M1 tended to generate positive results that were not confirmed by M2, M3, or both (see discordant pairs in Supplemental Table S2; https://doi.org/ 10.3168/jds.2018-15889). The discrepancy between M1 and M2 could result from the storage of milk at $-80^{\circ} \mathrm{C}$ after running M1 and before running M2. As M2 relies on a pre-enrichment culture step, any loss of viability could result in an increased Ct. However, $-80^{\circ} \mathrm{C}$ freezing and storage should be less deleterious than storage at $-20^{\circ} \mathrm{C}$, which had been found to diminish both $M$. agalactiae and $M$. mycoides ssp. capri viability over time (Amores et al., 2010). These discrepancies further demonstrate that the DNA extraction protocol (i.e., with the best balance between good extraction yield, removal of inhibitors, and absence of contamination) is critical when diagnosing CA by PCR, as already indicated (Tatay-Dualde et al., 2015).

The rtPCR results were interpreted using a first intention Ct cut-off of 40 (i.e., all samples yielding a 
Table 1. Real-time PCR method comparison 2 by 2 for the 2 Mycoplasma targets (Mycoplasma mycoides cluster and Mycoplasma agalactiae)

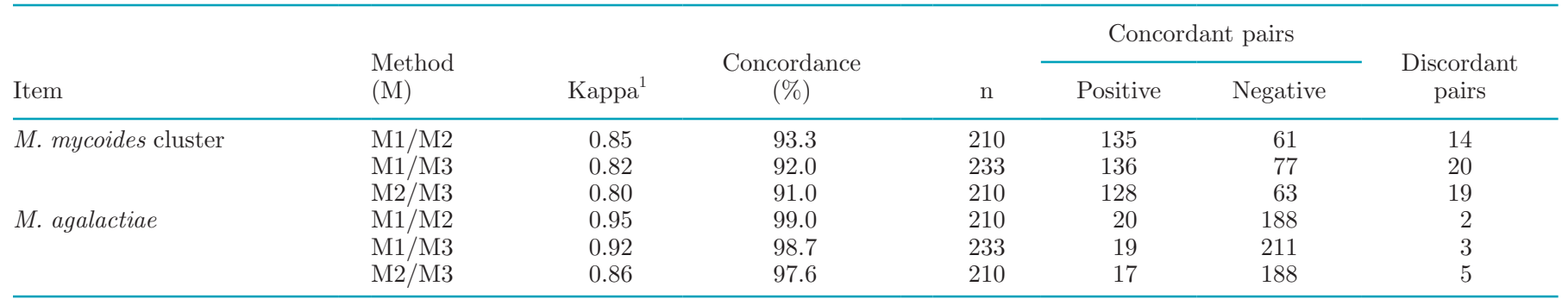

${ }^{1}$ Method agreement was calculated using Cohen's kappa coefficient.

Ct $>40$ were considered negative). However, 8 and 5 samples that were positive with M2 $(\mathrm{Ct} \leq 40)$, for M. mycoides cluster (Ct of 37.3, 38.1, 38.3, 39.4 twice, and 40 for 3 samples) and M. agalactiae (Ct of 35.8, $38.0,38.1,38.4$, and 39.3), respectively, were designated negative by culture. These discrepancies emphasize the difficulty of interpreting Ct values between 38 and 40 as these are most certainly doubtful, nonreproducible positive results, resulting from the potential presence of DNA without any living mycoplasma. Hence, any samples with a Ct higher than 38 must be interpreted with caution. The clinical relevance of strongly positive rtPCR samples is generally accepted by the clinicians, but it remains unclear what to do with weakly positive samples (Ct > 38), especially in the absence of clinical signs. Whether cultures or PCR are better adapted for CA-agent detection in BTM with low expected mycoplasmal loads is still controversial (Ariza-Miguel et al., 2012; Tatay-Dualde et al., 2015). Setting-up a cut-off for $\mathrm{Ct}$ interpretation is always difficult and should be adapted as a function of the epidemiological context and the purpose of detection (control versus eradication). In the present study, in which all the samples came from endemic areas, a combination of M1 and M2 would be ideal because the presence of living mycoplasma in the sample would result in a lower $\mathrm{Ct}$ with M2 than with M1, due to the pre-amplification step. At least, it would be advisable that any samples with $38<\mathrm{Ct} \leq 40$ using a direct DNA extraction should be re-analyzed using a pre-amplification of the target by a short culture time before DNA extraction. Furthermore, the rtPCR assays used here involve 3 detection probes and multiplex PCR are known to be potentially biased by preferential amplification or high background fluorescence when high-signal intensity dyes like FAM are used. The limit of detection (LOD) in raw milk, using the rtPCR assay, was shown to be $350 \mathrm{cfu} / \mathrm{mL}$ for both the M. mycoides cluster and $M$. agalactiae, with respective $\mathrm{Ct}$ of $38.9 \pm 0.5$ and $38.4 \pm 0.7$ (Becker et al., 2012). This LOD is a LOD of the overall method and not of the rtPCR alone; it takes the DNA extrac- tion step into account, which is hard to standardize due to the complex composition of raw milk samples and the presence of potential polymerase inhibitors.

If in second-line analysis, all samples with $\mathrm{Ct}>38$ were considered negative, then discrepancies between the rtPCR and culture results were only detected in 2 out of the 128 to 135 positive samples $(<2 \%)$. For these 2 samples, the $\mathrm{Ct}$ using M2 were 35.8 (M. agalactiae) and 37.3 (M. mycoides cluster), respectively. The most probable reason for these discrepancies could be a loss of mycoplasma viability resulting in a failure to regrow them (notably because the preculture, corresponding to a $\mathrm{Ct}$ of 37.3 , was accidentally frozen before being plated).

Overall, our analyses demonstrate the feasibility of using fresh, frozen milk or a FTA Elute card for the rtPCR assay without any significant loss of information and for all mycoplasma (sub)species involved in caprine CA. The BTM sampling and shipment could be simplified by using the FTA Elute card. However, DNA extraction from FTA cards requires a specific treatment protocol in a competent laboratory. The few samples that were positive on FTA membranes but negative with other techniques (see discordant pairs in Supplemental Table S2; https://doi.org/10.3168/ jds.2018-15889) could have resulted from the potential air-contamination of membranes while drying in the incubator, which suggests a need for caution if the FTA membrane were to be spotted next to the milk tank.

In conclusion, even if the methods are interchangeable for large numbers of analyses, any questionable result ( $\mathrm{Ct}>38$ or odd shape of the fluorescence curve) will require a new analysis as the consequence of a single positive result can be important for health herd management.

\section{Kinetics of Excretion of Mycoplasmas in BTM Over Time}

Potential minor discrepancies between the rtPCR methods were taken into consideration by further ana- 
lyzing milk positivity for mycoplasmas over time using method M2 only, which was conducted on all except a few samples. Figure 1 summarizes the different excretion patterns that were obtained in each herd for which milk was either negative (no bars) or positive (black bars) for one of the CA etiological agents. Three patterns were observed: always negative herds (2/20; PC9 and RA10); occasionally positive herds, that is, herds positive up to 6/24 mo (5/20; RA1, RA2, RA5, RA9, and PC4); and frequently positive herds, that is, herds positive over 6/24 mo (13/20; RA3, RA4, RA6, RA7, and RA8 and PC1, PC2, PC3, PC5, PC6, PC7, PC8, and PC10). Some of the frequently positive herds showed very variable results, with alternating positive and negative results (for instance, RA7). The species of mycoplasma had no effect (Table 2) on the excretion patterns and there was no relationship between the excretion pattern and the level of excretion (as estimated from the rtPCR Ct with M1, data not shown). By selecting herds with known histories of mycoplasmoses, we certainly biased the analyses as positive BTM would be expected over time due to the persistence of mycoplasmas. Hence for the 2 always negative herds, more specific monitoring, including other types of samples, would be of interest to confirm the absence of mycoplasmas and demonstrate the feasibility of mycoplasmosis eradication.

It seems that breeding factors (including drying periods, batching stress, food stress, and so on) did not influence the patterns of excretion and no correlation was present between excretion variation and the average tank cell count (Supplemental Table S1; https:/ /doi.org/10.3168/jds.2018-15889). The 2 always negative herds were both of enclosed Saanen breed but PC9 was larger than RA10 (550 vs. 150) with a higher cull rate (35 vs. $20 \%$ ). Some negative herds became positive during the 2 to 3 mo following onset of the new lactating period (Figure 1), which could correspond to an activation of latent infections at the beginning of lactation, as already suggested (Nicholas et al., 2008). However, any definitive conclusions are precluded by the small number of observations. This result is comparable to that of a previous study where the authors failed to establish a clear relationship between $M$. agalactiae excretion in milk and stages in sheep milk production (Ariza-Miguel et al., 2012).

The alternatively positive/negative BTM value could result from the intermittent excretion of mycoplasmas by different infected goats within the herd, a phenomenon well known for CA in sheep (Ariza-Miguel et al., 2012). The excretion measured from BTM samples is certainly underestimated as the mycoplasmal load in the tank will be diluted, depending on the number of excreting animals in the herd and the individual levels of excretion. In consequence, the results obtained by BTM sampling at a single time point could be below the level of detection. Hence, a single sample is insufficient to assess the risk of circulation/presence of mycoplasmas in a herd and several samples will be required over time. The frequency of such sampling has yet to be determined. No particular breeding period was identified here as being more favorable for sampling.

\section{Persistence of the Same (Sub)Species and Clone Within a Herd}

Detailed analysis of the isolates collected over this 2-yr longitudinal study revealed that they usually belonged to the same (sub)species within a herd (Table 2 ). This confirms that CA mycoplasmas can be highly persistent within a herd, up to $2 \mathrm{yr}$, as previously suggested (Corrales et al., 2007). In some herds, the combined circulation of different (sub)species was observed but most of the time one (sub)species was dominant. For example, in RA7, although up to 4 (sub)species were detected during the 2-yr study, the dominant one was $M$. agalactiae (isolated 9 mo out of 24). Interestingly, the RA7 herd was one of the smaller herds with a great external renewal rate. The observed diversity could result from the introduction of several new animals into the herd.

Interestingly, the relative proportions of the different (sub)species differed slightly from those observed in clinical cases of CA in France (Poumarat et al., 2014). Indeed, although M. mycoides ssp. capri and $M$. capricolum ssp. capricolum appeared to be the most recurrent (each present in 11/20 herds), M. agalactiae and $M$. putrefaciens were also frequently isolated $(4 / 20$ and $10 / 20$, respectively) even though these subspecies are usually underrepresented in clinical cases. However, this observation must be interpreted with caution as only 20 herds were investigated here.

Seven frequently positive herds were selected, and the corresponding isolates, which belonged to the same dominant (sub)species, were subtyped by PFGE (Figure 2). The diversity within a given herd is usually low and the same clone persists for months whatever the (sub)species. It should be noted that $M$. agalactiae was much less variable than subspecies in the $M$. mycoides cluster, as already reported (Nicholas et al., 2008). The PFGE patterns between herds were not identical.

The present results are consistent with those of a previous study, in which the persistence of the same $M$. mycoides ssp. capri-PFGE profile for 8 mo was demonstrated in the BTM from a herd that had undergone 


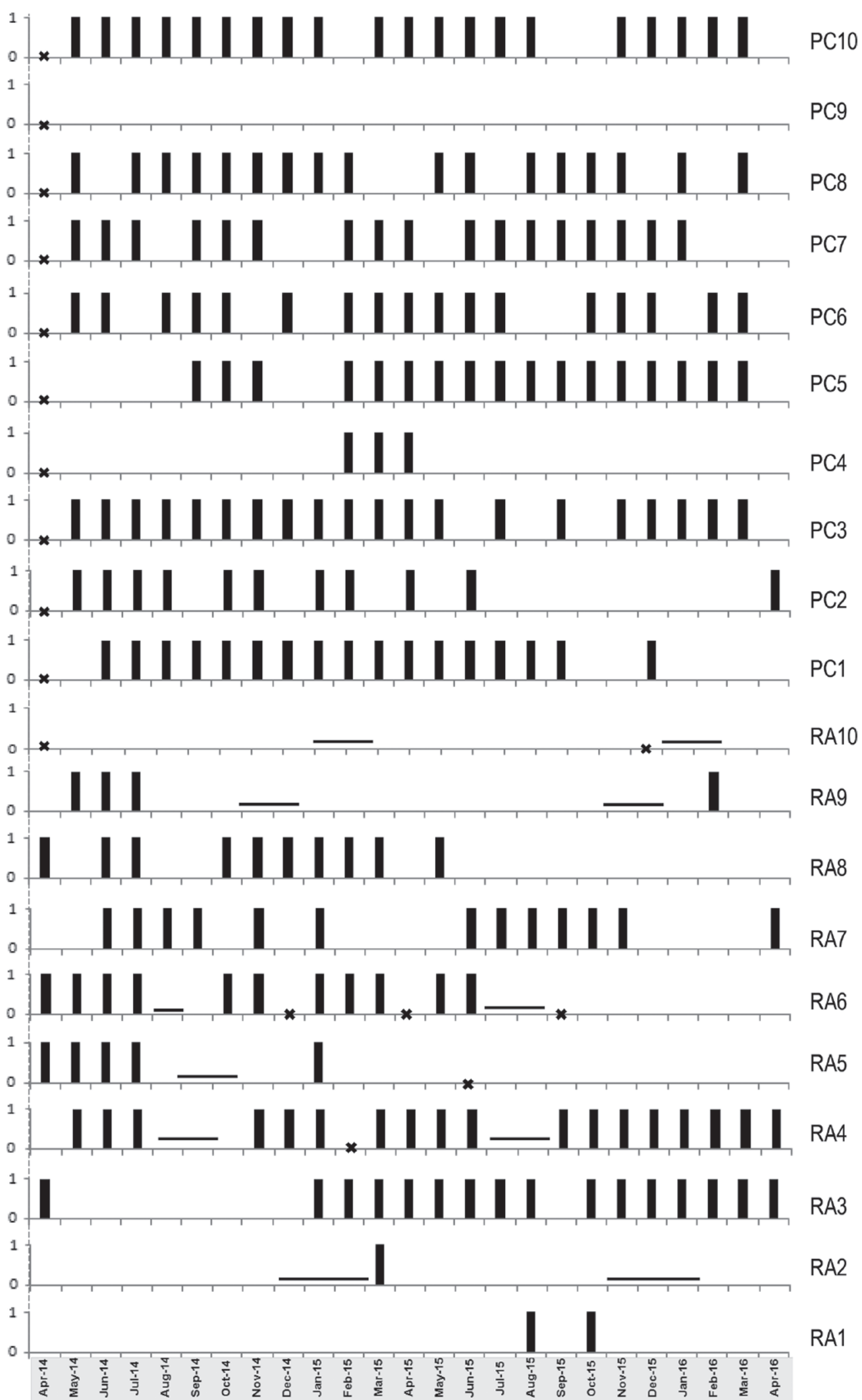

Figure 1. Excretion patterns of the 20 herds over time. The herd name is listed on the right of the panel. PC = Poitou-Charentes-Vendée; $\mathrm{RA}=$ Rhône-Alpes. Positive, monthly milks are indicated by black bars. The $\mathrm{x}$ indicates missing samples, and the bold horizontal lines indicate drying periods. 
Table 2. Recurrence of Mycoplasma species isolation per herd and herd categorization

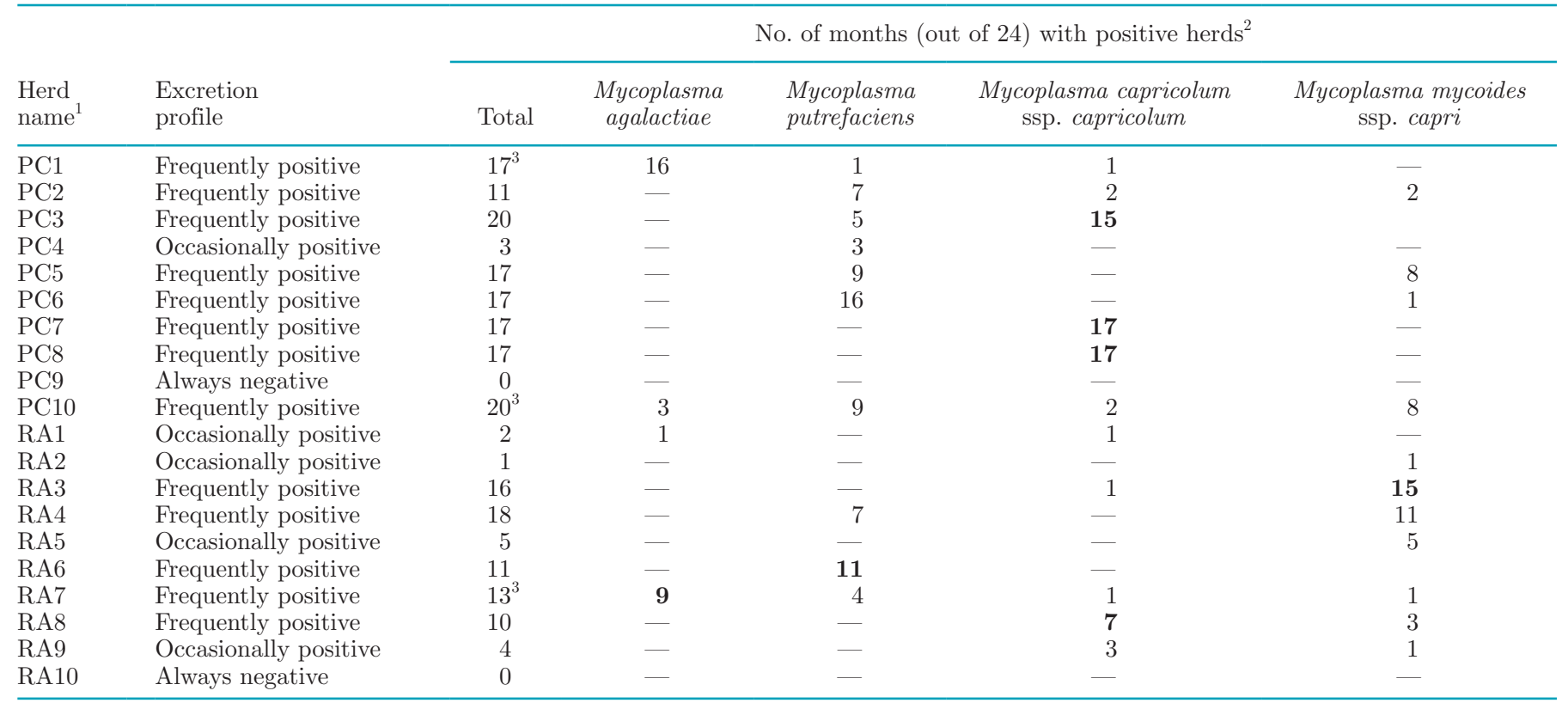

${ }^{1} \mathrm{PC}=$ Poitou-Charentes-Vendée; RA = Rhône-Alpes.

${ }^{2}$ Herds were considered positive when one of the targeted species was detected by method 2. Figures in bold indicate isolates selected to be analyzed by pulsed-field gel electrophoresis.

${ }^{3}$ Indicates when 1 or 2 bulk tank milk samples of these herds contained both M. agalactiae and another (sub)species (M. capricolum ssp. capricolum, M. mycoides ssp. capri, or M. putrefaciens).

a 2-mo acute clinical outbreak (Tardy et al., 2007). In France, the most extreme situation of mycoplasma persistence concerns the presence of $M$. agalactiae isolates, belonging to the same genetic subtype, for more than 35 yr now in ovine breeds of the Pyrénées-Atlantiques region, despite control efforts (Nouvel et al., 2012).

\section{BTM Surveillance as a Measure to Detect CA-Infected Herds}

Bulk tank milk sampling is considered noninvasive and cost effective; it requires little time and has been proposed for surveillance programs relying on PCR or ELISA tests for controlled caprine diseases such as Q-fever, caprine arthritis encephalitis, and foodborne disease due to Staphylococcus aureus (Spanu et al., 2013; Nagel-Alne et al., 2015; Van den Brom et al., 2015). Bulk tank milk sampling has also been recently validated for use in the control of an important bovine mycoplasma, Mycoplasma bovis, by identifying herds from which purchase of animals could increase the biosecurity risk of introducing Mycoplasma bovis into noninfected herds (Parker et al., 2017). The oftenrecurring question in these different studies is how to determine the test cut-off and be able to distinguish infected from noninfected herds, especially in goat herds that are expected to be free of the disease (e.g., eradication program or area of low prevalence). In the present study, we were challenged not only with the intermittent excretion of mycoplasmas by animals and, at the same time, their long-term persistence within a herd but also the need for reliable processing of the BTM sample. The following recommendations can be derived from our results with regard to using BTM to survey the status of herds with respect to mycoplasmosis: there is a need for (1) an optimized DNA extraction method, (2) a sensitive detection technique with a consensus cut-off for interpretation, and (3) several sampling points over time. This frequency of sampling has yet to be defined and might be even more critical for herds expected to be mycoplasma free.

\section{ACKNOWLEDGMENTS}

The authors are grateful to the breeders and professional organizations who participated in this study and to la Région Nouvelle Aquitaine, le département des Deux Sèvres, le Pôle d'Expérimentation et de Progrès Caprin Rhône Alpes, le Groupements de Défense Sanitaire Rhône-Alpes, and la groupe coopératif AGRIcole et agroALimentaire for their financial support. We thank Adélie Colin and Catherine Mottet (UMR Mycoplasmoses des Ruminants) for their participation in laboratory analyses. 
A Percentage of similarity

BamHI

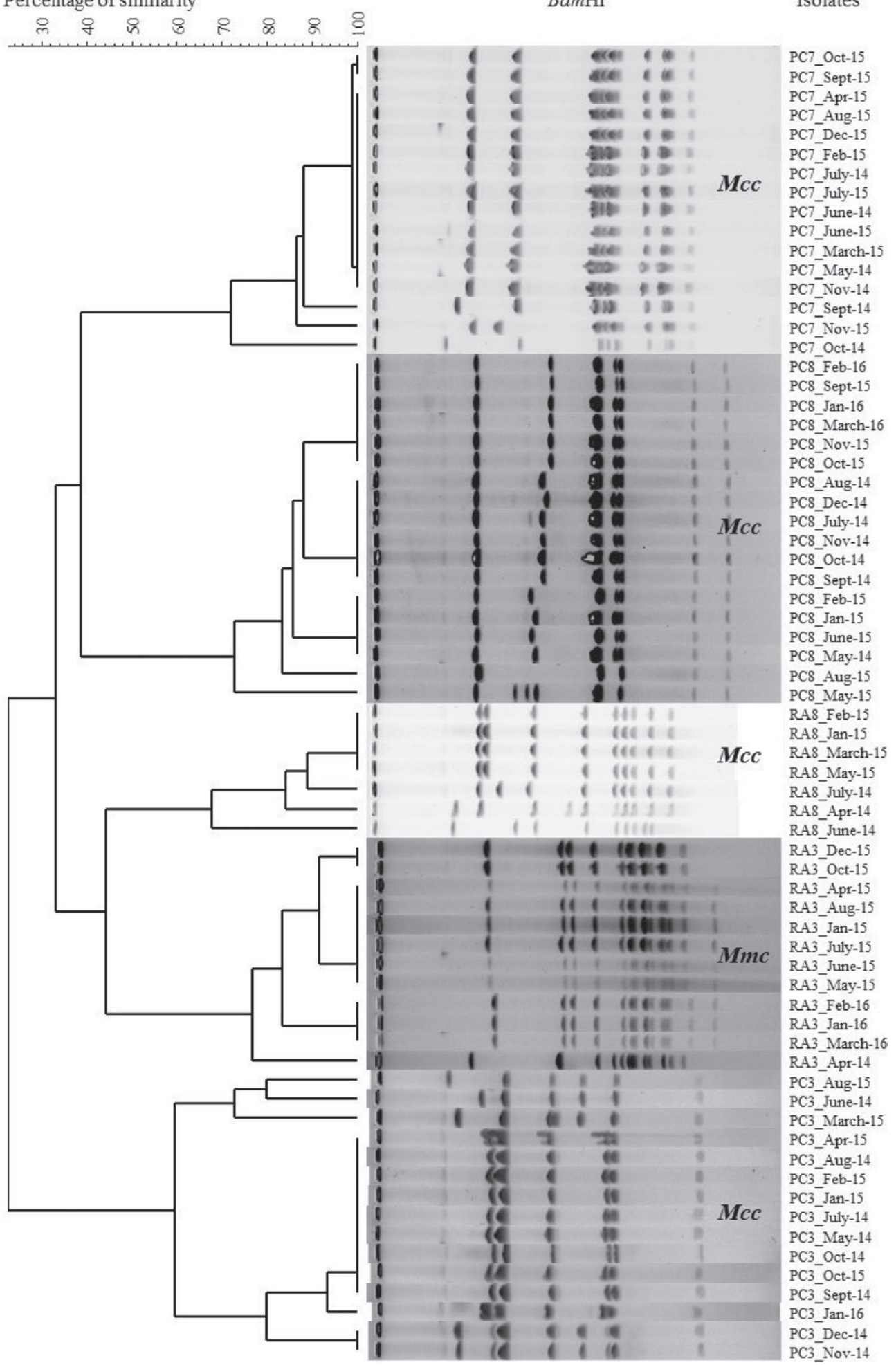

Figure 2. Dendrograms displaying Mycoplasma capricolum ssp. capricolum or Mycoplasma mycoides ssp. capri BamHI (A) and Mycoplasma agalactiae or Mycoplasma putrefaciens MluI (B) pulsed-field gel electrophoresis (PFGE) profiles. Sixty-eight M. capricolum ssp. capricolum or M. mycoides ssp. capri clones isolated from PC3, PC7, PC8, RA3, and RA8 herds (A) and 16 M. putrefaciens or M. agalactiae clones isolated from RA6 and RA7 herds (B) were analyzed, where PC $=$ Poitou-Charentes-Vendée and RA = Rhône-Alpes. Cluster analyses are based on the PFGE profiles using the Dice coefficient and unweighted pair group method with arithmetic mean. The resulting degree of similarity is indicated on the scale on the left. Clones are characterized by the herd name, month, and year of isolation. Mcc = Mycoplasma capricolum ssp. capricolum; $M m c=$ Mycoplasma mycoides ssp. capri; Mput = Mycoplasma putrefaciens; Maga = Mycoplasma agalactiae 


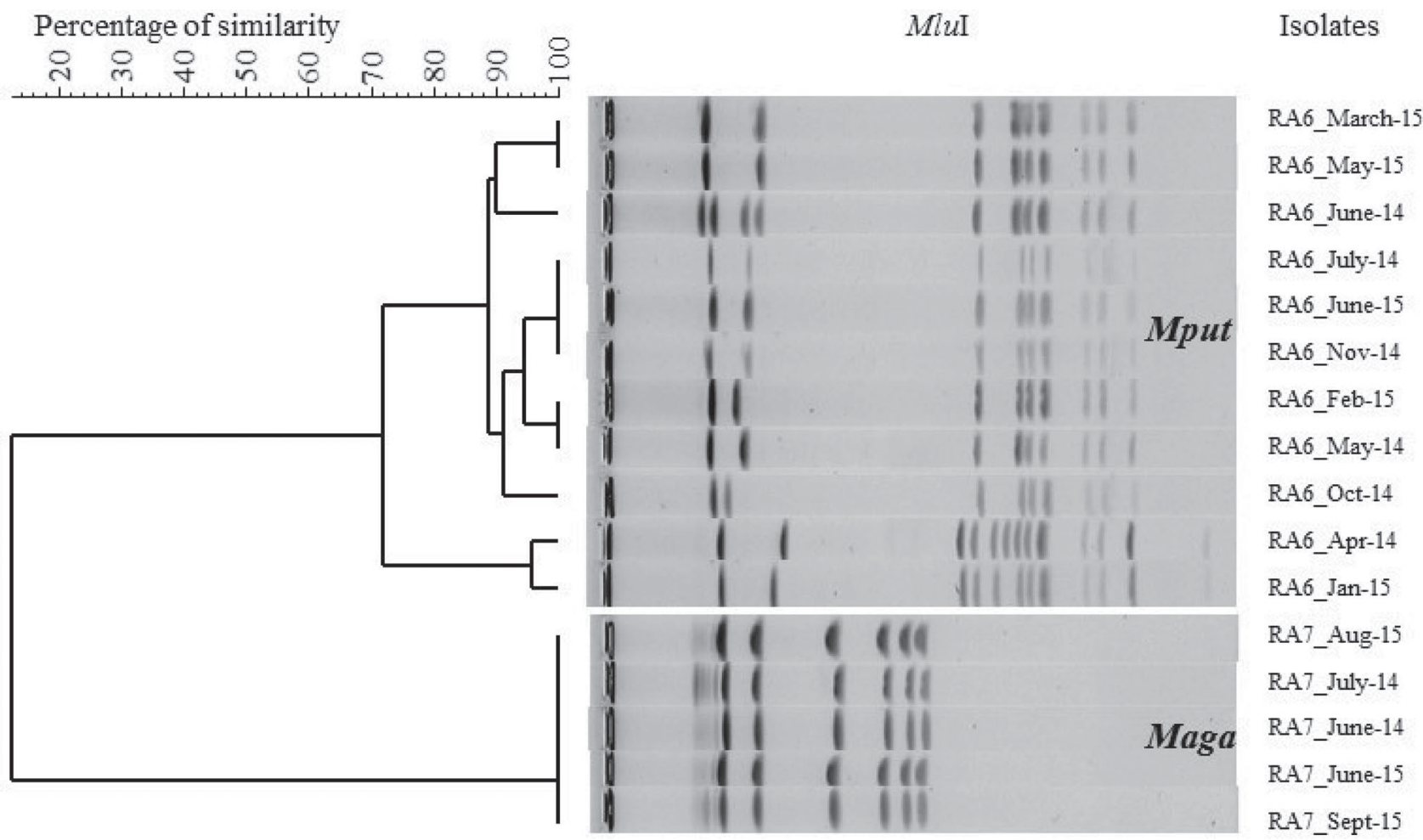

Figure 2 (Continued). Dendrograms displaying Mycoplasma capricolum ssp. capricolum or Mycoplasma mycoides ssp. capri BamHI (A) and Mycoplasma agalactiae or Mycoplasma putrefaciens MluI (B) pulsed-field gel electrophoresis (PFGE) profiles. Sixty-eight M. capricolum ssp. capricolum or M. mycoides ssp. capri clones isolated from PC3, PC7, PC8, RA3, and RA8 herds (A) and 16 M. putrefaciens or M. agalactiae clones isolated from RA6 and RA7 herds (B) were analyzed, where PC = Poitou-Charentes-Vendée and RA = Rhône-Alpes. Cluster analyses are based on the PFGE profiles using the Dice coefficient and unweighted pair group method with arithmetic mean. The resulting degree of similarity is indicated on the scale on the left. Clones are characterized by the herd name, month, and year of isolation. Mcc = Mycoplasma capricolum ssp. capricolum; Mmc = Mycoplasma mycoides ssp. capri; Mput = Mycoplasma putrefaciens; Maga = Mycoplasma agalactiae.

\section{REFERENCES}

Agnone, A., M. La Manna, G. Sireci, R. Puleio, A. Usticano, U. Ozdemir, R. A. J. Nicholas, V. Chiaracane, F. Dieli, V. Di Marco, and G. R. Loria. 2013. A comparison of the efficacy of commercial and experimental vaccines for contagious agalactia in sheep. Small Rumin. Res. 112:230-234.

Al-Momani, W., R. A. Nicholas, and M. N. Abo-Shehada. 2008. Risk factors associated with Mycoplasma agalactiae infection of small ruminants in northern Jordan. Prev. Vet. Med. 83:1-10.

Amores, J., A. Sanchez, A. G. Martin, J. C. Corrales, A. Contreras, and C. de la Fe. 2010. Viability of Mycoplasma agalactiae and Mycoplasma mycoides ssp. capri in goat milk samples stored under different conditions. Vet. Microbiol. 145:347-350.

Ariza-Miguel, J., D. Rodriguez-Lazaro, and M. Hernandez. 2012. A survey of Mycoplasma agalactiae in dairy sheep farms in Spain. BMC Vet. Res. 8:171.

Becker, C., F. Ramos, E. Sellal, S. Moine, F. Poumarat, and F. Tardy. 2012. Development of a multiplex real-time PCR for contagious agalactia diagnosis in small ruminants. J. Microbiol. Methods 90:73-79.

Bergonier, D., and F. Poumarat. 1996. Contagious agalactia of small ruminants: Epidemiology, diagnosis and control. Rev. Off. Int. Epizoot. 15:1431-1475.

Corrales, J. C., A. Esnal, C. De la Fe, A. Sanchez, P. Assuncao, J. B. Poveda, and A. Contreras. 2007. Contagious agalactia in small ruminants. Small Rumin. Res. 68:154-166.
Gomez-Martin, A., J. Amores, A. Paterna, and C. De la Fe. 2013. Contagious agalactia due to Mycoplasma spp. in small dairy ruminants: Epidemiology and prospects for diagnosis and control. Vet. J. 198:48-56.

Gomez-Martin, A., C. De la Fe, J. Amores, A. Sanchez, A. Contreras, A. Paterna, A. J. Buendia, and J. C. Corrales. 2012. Anatomic location of Mycoplasma mycoides ssp. capri and Mycoplasma agalactiae in naturally infected goat male auricular carriers. Vet. Microbiol. 157:355-362.

Landis, J. R., and G. G. Koch. 1977. The measurement of observer agreement for categorical data. Biometrics 33:159-174.

Nagel-Alne, G. E., P. S. Valle, R. Krontveit, and L. S. Solverod. 2015. Caprine arthritis encephalitis and caseous lymphadenitis in goats: Use of bulk tank milk ELISAs for herd-level surveillance. Vet. Rec. 176:173.

Nicholas, R. A. J., R. D. Ayling, and L. McAuliffe. 2008. Chapter 8: Contagious Agalactia. Pages 98-113 in Mycoplasma Diseases of Ruminants. CABI, Oxfordshire, UK.

Nicholas, R. A. J., and S. E. Baker. 1998. Recovery of mycoplasmas from animals. Pages 37-44 in Mycoplasma Protocols. Vol. 104. R. J. Miles and R. A. J. Nicholas, ed. Humana Press Inc., Totowa, NJ.

Nouvel, L.-X., M. S. Marenda, M. D. Glew, E. Sagne, P. Giammarinaro, F. Tardy, F. Poumarat, R. Rosengarten, and C. Citti. 2012 Molecular typing of Mycoplasma agalactiae: Tracing Europeanwide genetic diversity and an endemic clonal population. Comp. Immunol. Microbiol. Infect. Dis. 35:487-496. 
Parker, A. M., J. K. House, M. S. Hazelton, K. L. Bosward, J. M. Morton, and P. A. Sheehy. 2017. Bulk tank milk antibody ELISA as a biosecurity tool for detecting dairy herds with past exposure to Mycoplasma bovis. J. Dairy Sci. 100:8296-8309.

Poumarat, F., A. V. Gautier-Bouchardon, D. Bergonier, E. Gay, and F. Tardy. 2016. Diversity and variation in antimicrobial susceptibility patterns over time in Mycoplasma agalactiae isolates collected from sheep and goats in France. J. Appl. Microbiol. 120:1208 1218.

Poumarat, F., N. Jarrige, and F. Tardy. 2014. Purpose and overview of results of the Vigimyc Network for the epidemiological surveillance of mycoplasmoses in ruminants in France. EuroRéférence $12: 24-28$.

Poumarat, F., B. Perrin, and D. Longchambon. 1991. Identification of ruminant mycoplasmas by dot immunobinding on membrane filtration (MF-dot). Vet. Microbiol. 29:329-338.

Prats-van der Ham, M., J. Tatay-Dualde, C. Ambroset, C. De la Fe, and F. Tardy. 2018. The moderate drift towards less tetracycline-susceptible isolates of contagious agalactia causative agents might result from different molecular mechanisms. Vet. Microbiol 220:39-46.

Spanu, V., C. Scarano, S. Virdis, S. Melito, C. Spanu, and E. P. De Santis. 2013. Population structure of Staphylococcus aureus isolated from bulk tank goat's milk. Foodborne Pathog. Dis. 10:310-315.

Tardy, F., P. Mercier, M. Solsona, E. Saras, and F. Poumarat. 2007. Mycoplasma mycoides ssp. mycoides biotype large colony isolates from healthy and diseased goats: Prevalence and typing. Vet. Microbiol. 121:268-277.

Tatay-Dualde, J., A. Sanchez, M. Prats-van der Ham, A. Gomez-Martin, A. Paterna, J. C. Corrales, C. de la Fe, A. Contreras, and J. Amores. 2015. Sensitivity of two methods to detect Mycoplasma agalactiae in goat milk. Ir. Vet. J. 68:21.

Van den Brom, R., I. Santman-Berends, S. Luttikholt, L. Moll, E. Van Engelen, and P. Vellema. 2015. Bulk tank milk surveillance as a measure to detect Coxiella burnetii shedding dairy goat herds in the Netherlands between 2009 and 2014. J. Dairy Sci. 98:38143825 